\title{
Synthesis of some hydroxamic acidsLinked-naturalsamino acid
}

\author{
Marzough Aziz DagerAlbalawi ${ }^{1 *}$ \\ ${ }^{I}$ Department of Chemistry, University college-Alwajh, University of Tabuk, Saudi Arabia
}

Abstract: A series of amino acid derivative hydroxamic acid have been synthesized as promising compounds for further biological activity. These compounds were fully characterized by spectroscopic techniques including; $1 H, 13 C, D E P T 135$ and HRMS.

Keyswords:Amino acid, Hydroxamic acid, Sulfonamides.

\section{Introduction}

Since their discovery by Wahlroos and Virtanen (1) in 1959, and over the past decades, the chemistry and biochemistry of hydroxamic acids and their derivatives have attracted considerable attention, due to their pharmacological, toxicological and pathological properties. Recently, the compounds containing hydroxamic acid functionality, have been reported to exhibit multifarious pharmacological effects including antioxidant, [1] , anticancer, [2,3] , antibacterial agents, [4] , antifungal, [5] , growth factors, [6] , anti-melanogenic, [7] , antimalarial,[8], enzyme inhibitors, [9] , rare earth mineral collectors, [10] , and reagents for solvent extraction and spectrophotometric determination of metals , [11] .A number of hydroxamic acid derivatives have also been found to inhibit several important enzymes i n cellular systems, including FeII and MnII E. coli methionine aminopeptidase, [12], $\gamma$-lactum based histone deacetylase (HDACs),[13] , and matrix metalloproteinases, [14, 15].In view of the above applications, the present work relates to the synthesis and characterization of new hydroxamic acids.

\section{Results And Discussion}

In present investigation the synthetic approach was achieved in three steps as shown in Scheme1.Initially commercially available amino acid $\mathbf{1}$ has been protected withphenylsulfonyl chloride in the presence of one equivalent of sodium hydroxideto afford compound 2.Compound 2, when subjected to condensation with paraformaldehyde and para toluene sulfonic acid as catalyst gave the corresponding 1,3-oxazolidin -5-ones $\mathbf{3}$. The cleavage of the latter with the hydroxylamine hydrochloride in methanol led to the $\mathrm{N}$ - phenylsulfonyl -2amino hydroxamic acids4as shown in the scheme 1 .<smiles>[2H]C(N)C(=O)O</smiles>
a, $\mathrm{R}=$ isoPr
b, $R=$ Phenyl
c, $\mathrm{R}=$ isoBut
d, $\mathrm{R}=$ sec-But

scheme 1: Synthetic route for the synthesis of hydroxamic acids a-d 
In each step, progress of the reaction was monitored by TLC (Thin layer chromatograpy). The ${ }^{1} \mathrm{H}$ NMR spectra of hydroxamic acids under investigation show the characteristic singlet of the proton of the hydroxyl group in the region 11-11.5 ppm. A broad signal in the region 7.2-8.22 ppm which evidently belonged to the Ar$\mathrm{H}$ and $\mathrm{NH}$ protons of the hydroxylamine unit. The ${ }^{13} \mathrm{C}-\mathrm{NMR}$ spectra exhibit absorption signal due to carbonyl, $\mathrm{C}=\mathrm{O}$ carbon nearby $165 \mathrm{ppm}$. The chemical shifts of aromatic carbon appear in the region 124-138 ppm. Beside these signals, a singlet near2 $1 \mathrm{ppm}$ and a singlet at $171 \mathrm{ppm}$ appeared which correspond to the carbon atom of alkyl group and carboxy group respectively.DEPT 135 spectrum indicates that the methylene carbon appears at $24.45,25.28$ and $37.24, \mathrm{CH}$ carbons are noticed in the region between $127.7 \mathrm{ppm}$ to $134.8 \mathrm{ppm}$.

\section{Experimental}

All reactions were performed under an argon atmosphere and were monitored by thin-layer chromatography (TLC) Merck 60 F-254 silica-gel plates (layer thickness $0.25 \mathrm{~mm}$ ). Column chromatography was performed on silica gel (70-230 mesh) using ethyl acetate and cyclohexane mixture as eluents. Melting temperatures were determined on an Electrothermal 9002 apparatus and were reported uncorrected. NMR spectra were recorded on a Bruker AC-300 spectrometer at $300 \mathrm{MHz}\left({ }^{1} \mathrm{H}\right)$ and $75 \mathrm{MHz}\left({ }^{13} \mathrm{C}\right)$. All chemical shifts were reported as $\mathbf{d}$ values $(\mathrm{ppm})$ relative to internal tetramethylsilane. High-resolution mass spectra (HRMS) were recorded on a matrix-assisted laser desorption=ionization time-of-flight (MALDI-TOF) Perspective Biosystems Voyager DE-STR instrument.

Synthesis:

General procedure for synthesis of sulfonamide protected acids:

The starting materials 2a-d were prepared according to [16 ].

$\boldsymbol{N}$-phenylsulfonyl-L-valine $\quad$ (2a). $\quad$ Prepared from $\quad$ commercial andPtoluenesulfonylchlorideCrystallization from ether afforded the productas a white solid (yield $80 \%$ ); ${ }^{1} \mathrm{H}$ NMR (300 MHz, methanol-d4) $\delta 0.90(\mathrm{~d}, J=6.9 \mathrm{~Hz}, 3 \mathrm{H}), 0.93(\mathrm{~d}, J=6.9 \mathrm{~Hz}, 3 \mathrm{H}), 2.21(\mathrm{~m}, 1 \mathrm{H}), 3.54(\mathrm{~d}, J=$ $7.5 \mathrm{~Hz}, 1 \mathrm{H}), 7.62-7.78(\mathrm{~m}, 7 \mathrm{H})$.

$N$-phenylsulfonyl-L-phenylalanine (2b). Prepared from L-phenylalanine; $1 \mathrm{H}$ NMR (300 MHz, methanol- $d 4)$ $\delta 3.22(\mathrm{~d} J=14.7,2 \mathrm{H}), 4.87(\mathrm{t}, 1 \mathrm{H}), 7.32-7.98(\mathrm{~m}, 11 \mathrm{H}), 11.51(\mathrm{~s}, 1 \mathrm{H})$.

$N$-phenylsulfonyl-L-leucine (2c). Prepared from commercial L-isoleucine; ${ }^{1} \mathrm{H}$ NMR (300 MHz, methanol- $\left.d 4\right)$ $\delta 0.86(\mathrm{~d}, J=6.4 \mathrm{~Hz}, 6 \mathrm{H}), 1.68(\mathrm{~m}, 1 \mathrm{H}) 2.16(\mathrm{t}, 2 \mathrm{H}), 3.81(\mathrm{~m}, 1 \mathrm{H}), 7.61-7.87(\mathrm{~m}, 6 \mathrm{H}), 11.55(\mathrm{~s}, 1 \mathrm{H})$.

$\boldsymbol{N}$-phenylsulfonyl-L-isoleucine(2d). Prepared from L-leucine; ${ }^{1} \mathrm{H}$ NMR (300 MHz, methanol- $\left.d 4\right) \delta 0.85$ (m, $6 \mathrm{H}), 1.32(\mathrm{q}, 2 \mathrm{H}), 1.83(\mathrm{~m}, 1 \mathrm{H}), 3.77(\mathrm{~d}, J=9.2,1 \mathrm{H}), 7.61-7.88(\mathrm{~m}, 6 \mathrm{H}), 11.58(\mathrm{~s}, 1 \mathrm{H})$.

General procedure for synthesis ofsubstituted1,3- oxazolidin -5-ones:

The reactions were performed according to the litterature [17]. the synthesis of substituted 1,3- oxazolidin -5ones by stirring suspension of sulfonamides $\mathbf{2 a - d}$, toluene, paraformaldehyde and para toluene sulfonic acidas catalyst. It was reported that the reaction was completed at room temperature in a short duration of time with good yield of oxazolidin-5-one .

Synthesis of product 4-isopropyl-3-(phenylsulfonyl)oxazolidin-5-one $\underline{3 a}$ :The mixture of compound 2a , paraformaldehyde, Toluene and paratoluensulfonic acid as catalyst afforded the compound 3a.yield :65\% NMR ${ }^{1} \mathbf{H}:\left(300 \mathrm{MHz}, \mathrm{CDCl}_{3}\right) \delta: 0.98(\mathrm{~d}, 6 \mathrm{H}), 2.00(\mathrm{q}, 1 \mathrm{H}), 3.62(\mathrm{dd}, J=14.8,1 \mathrm{H}), 7.42-7.9(\mathrm{~m}, 7 \mathrm{H}) \cdot{ }^{13} \mathrm{C}:(75 \mathrm{MHz}$, $\left.\mathrm{CDCl}_{3}\right) \delta: 18.42,18.46,31.04,62.76(\mathrm{C}-\mathrm{N}), 94.11\left(\mathrm{CH}_{2}-\mathrm{O}\right), 127.28-136.00$ (6 C aromatic), $171.19(\mathrm{CO})$.

\section{Synthesis of product 4-benzyl-3-(phenylsulfonyl)oxazolidin-5-one $\underline{3 b}$ :}

The compound $\mathbf{3 b}$ was prepared as the same procedure as 3awich was obtained as white solid. Yield :70\%.NMR ${ }^{1} \mathbf{H}:\left(300 \mathrm{MHz}, \mathrm{CDCl}_{3}\right) \delta: 3.31(\mathrm{~d}, 2 \mathrm{H}, \mathrm{J}=5.6), 4.45(\mathrm{t}, 1 \mathrm{H}), 5.65(\mathrm{dd}, J=9.2,2 \mathrm{H}), 7.22-7.94(\mathrm{~m}, 10 \mathrm{H}) .{ }^{13} \mathrm{C}$ : $\left(75 \mathrm{MHz}, \mathrm{CDCl}_{3}\right) \delta: 37.24,58.54,79.83\left(\mathrm{CH}_{2}-\mathrm{O}\right), 127.49-136.13$ (12 C aromatic), 171.39(CO);DEPT 135: (300 $\left.\mathrm{MHz}, \mathrm{CDCl}_{3}\right) \delta$ :

Synthesis of product 4-isobutyl-3-(phenylsulfonyl)oxazolidin-5-one $\underline{3 \mathrm{c}}$ :

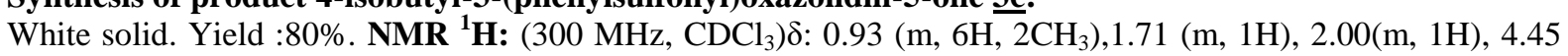
$(\mathrm{t}, 1 \mathrm{H}), 5.31(\mathrm{~d}, J=14.6,1 \mathrm{H}), 5.7(\mathrm{~d}, J=14.4,1 \mathrm{H}), 7.61-7.92(\mathrm{~m}, 5 \mathrm{H})$.

${ }^{13} \mathrm{C}:\left(75 \mathrm{MHz}, \mathrm{CDCl}_{3}\right) \delta: 21.43,22.75,24.45,38.44,56.20,78.76\left(\mathrm{CH}_{2}-\mathrm{O}\right), 127.64-135.98$ (6 $\mathrm{C}$ aromatic), 172.249(CO).

Synthesis of product 4-sec-butyl-3-(phenylsulfonyl)oxazolidin-5-one $\underline{3 \mathrm{~d}}$ :

White solid: Yield 54\%. NMR ${ }^{1} \mathbf{H}:\left(300 \mathrm{MHz}, \mathrm{CDCl}_{3}\right) \delta: 1\left(\mathrm{~m}, 6 \mathrm{H}, 2 \mathrm{CH}_{3}\right), 1.44(\mathrm{~m}, 2 \mathrm{H}), 2.22(\mathrm{~m}, 1 \mathrm{H}), 4.46(\mathrm{~d}, J$ $=14.6,1 \mathrm{H}), 5.32(\mathrm{~d}, J=14.6,1 \mathrm{H}), 5.6(\mathrm{~d}, J=14.6,1 \mathrm{H}) 7.61-7.95(\mathrm{~m}, 5 \mathrm{H}) \cdot{ }^{13} \mathrm{C}:\left(75 \mathrm{MHz}, \mathrm{CDCl}_{3}\right) \delta: 11.46$, $14.93,25.28,37.75,61.76,79.56\left(\mathrm{CH}_{2}-\mathrm{O}\right), 127.63-135.95$ (6 $\mathrm{C}$ aromatic), 171.03(CO). 
General procedure for the synthesis of Hydroxamic acid:

Synthesis of product (S)-N-hydroxy-3-methyl-2-(phenylsulfonamido)butanamide4a: White solid;m.p. 119$121{ }^{\circ} \mathrm{C}$; Yield62.5\%. ${ }^{1} \mathbf{H}-\mathbf{N M R}\left(\mathrm{CDCl}_{3}\right) \delta: 0.8\left(\mathrm{~m}, 6 \mathrm{H}, 2 \mathrm{CH}_{3}\right), 2(\mathrm{~m}, 1 \mathrm{H}), 7.43-8 . \overline{22}(\mathrm{~m}, 7 \mathrm{H}) ; 11.3(\mathrm{~s}, 1 \mathrm{H}$, $\mathrm{OH}) ;{ }^{13} \mathrm{C}-\mathrm{NMR}\left(\mathrm{CDCl}_{3}\right) \quad \delta: \quad 16.71, \quad 17.48, \quad 31.37, \quad 61.12(2 \mathrm{C}), \quad 127.21(2 \mathrm{C}), \quad 127.28, \quad 132.75, \quad 139.38$, 171.09(CO).DEPT $135 \delta$ : 18.42, 18.46, 31.4, 62.76, 127.64, 128.21, 128.93, 129.89, 134.31. (MALDI-TOF) calculated forC $\mathrm{C}_{15} \mathrm{H}_{16} \mathrm{~N}_{2} \mathrm{O}_{4} \mathrm{~S}[\mathrm{M}]^{+}: \mathrm{C}_{11} \mathrm{H}_{16} \mathrm{~N}_{2} \mathrm{O}_{4} \mathrm{~S}[\mathrm{M}]^{+}: 272.08308$ Found: 272.08221

Synthesis of product(S)-N-hydroxy-3-phenyl-2-(phenylsulfonamido)propanamide $\underline{4 \mathrm{~b}}$ :

NMR ${ }^{1} \mathbf{H}:\left(300 \mathrm{MHz}, \mathrm{CDCl}_{3}\right) \delta: 3.25(\mathrm{dd}, \mathrm{J}=14.8, \mathrm{~J}=5.6 \mathrm{~Hz}, 2 \mathrm{H}), 4.13(\mathrm{t}, 1 \mathrm{H}), 7.20-8 . \overline{16}(\mathrm{~m}, 12 \mathrm{H}), 11.35(\mathrm{~s}, 1 \mathrm{H}$, $\mathrm{OH}) .{ }^{13} \mathrm{C}:\left(75 \mathrm{MHz}, \mathrm{CDCl}_{3}\right) \delta: 37.30\left(\mathrm{CH}_{2}\right), 57.23,126.81-138.16$ (12 C aromatic), 171.40(CO). DEPT $135 \delta$ : 37.24 $\left(\mathrm{CH}_{2}\right)$, 58.54, 127.49, 127.73, 128.83, 129.89, 129.94,134.26. HRMS (MALDI-TOF) calculated forC $\mathrm{C}_{15} \mathrm{H}_{16} \mathrm{~N}_{2} \mathrm{O}_{4} \mathrm{~S}[\mathrm{M}]^{+}$: 320.08307 Found: 305.08162 .

Synthesis of product (S)-N-hydroxy-4-methyl-2-(phenylsulfonamido)pentanamide $\underline{4 c}$ :

A yellowish solid, m.p: $153-156{ }^{\circ} \mathrm{C}$; Yield 61.8\%; ${ }^{1} \mathrm{H}$ NMR $\left(300 \mathrm{MHz}, \mathrm{CDCl}_{3}\right) \delta$ : $0 . \overline{98}\left(\mathrm{~d}, 6 \mathrm{H}, 2 \mathrm{CH}_{3}\right), 1.5(\mathrm{~m}$, $1 \mathrm{H}), 1.89(\mathrm{~m}, 1 \mathrm{H}), 3.88(\mathrm{t}, 1 \mathrm{H}), 7.56-8.22(\mathrm{~m}, 7 \mathrm{H}), 11.16(\mathrm{~s}, 1 \mathrm{H}, \mathrm{OH}) ;{ }^{13} \mathrm{C}:\left(75 \mathrm{MHz}, \mathrm{CDCl}_{3}\right) \delta: 21.45,21.98$, $24.45,38.44,56.20,124.70-128.9$ (5C), 173.12(CO).DEPT $135 \delta: 21.43,22.75,24.45\left(\mathrm{CH}_{2}\right), 38.44,56.20$, 127.46, 128.21, 129.9, 134.36. HRMS (MALDI-TOF) calculated forC $_{12} \mathrm{H}_{18} \mathrm{~N}_{2} \mathrm{O}_{4} \mathrm{~S}[\mathrm{M}]^{+}$: 286.09761Found: 286.09218 .

Synthesis of product (2S)-N-hydroxy-3-methyl-2-(phenylsulfonamido)pentanamide $\underline{4 d}$ : White solid: m.p:161-164 ${ }^{\circ} \mathrm{C}$.Yield 54\%. NMR ${ }^{1} \mathrm{H}:\left(300 \mathrm{MHz}, \mathrm{CDCl}_{3}\right) \delta: 0.93\left(\mathrm{~d}, 6 \mathrm{H}, 2 \mathrm{CH}_{3}\right), 1.55(\mathrm{~m}, 2 \mathrm{H}), 2.12(\mathrm{~m}, 1 \mathrm{H}), 3.78$ $(\mathrm{d}, J=14.5,1 \mathrm{H}), 7.61-8.11(\mathrm{~m}, 5 \mathrm{H}) \cdot{ }^{13} \mathrm{C}:\left(75 \mathrm{MHz}, \mathrm{CDCl}_{3}\right) \delta: 11.78,14.16,26.4,34.28,62.3,125.21-138.8(\mathrm{C}$ aromatic), 172.82(CO).DEPT $135 \delta: 11.46,14.83,25.28\left(\mathrm{CH}_{2}\right), 37.75,127.63,129.89,134.3$. HRMS (MALDITOF) calculated forC ${ }_{12} \mathrm{H}_{18} \mathrm{~N}_{2} \mathrm{O}_{4} \mathrm{~S}[\mathrm{M}]^{+}:$286.09872Found: 286.09213.

\section{Concusion}

We have developed a facile method to synthesize differents amino acid derived Hydroxamic acid. The reaction offers convenience, mild conditions and good to excellent yields. Evaluation of this product in biological activity is in progress.

\section{Acknowledgments:}

This work was supported by the Ministry of Higher Education of Saoudi Arabia

\section{Refrences}

[1]. M. Z. Koncic, M. Barbaric, I. Perkovic, B. Zorc, Molecules 2011, 16, 6232-6242.

[2]. D. K. Pal, S. Saha, J. Adv. Pharm. Tech. Res. 2012, 3, 92-99.

[3]. L. P. Tardibono Jr, M. J. Miller, Org. Lett. 2009, 11, 1575-1578.

[4]. H. Jahangirian, J. Haron, S. Silong, N. A. Yusof, K. Shameli, S. Eissazadeh, R. R. Moghaddam, B. Mahdavi, M. Jafarzade, J. Med. P. Res. 2011, 5, 4826-4831.

[5]. R. B. Hector, W. Lazo, J. Agric. Food Chem. 1996, 44, 1569-1571.

[6]. E. Nakagawa, T. Amano, N. Hirai, H. Iwamura, Phytochemistry 1995, 38, 1349-1354.

[7]. H. S. Rho, H. S. Baek, S. M. Ahn, J. W. Yoo, D. H. Kim, H. G. Kim, Bull. Korean Chem. Soc. 2009, 30, 475-482.

[8]. K. P. Holland, H. L. Elford, V. Bracchi, C. G. Annis, S. M. Schuster, D. Chakrabarti, Antimicrob. Agent. Chemother. 1998, 42, 2456-2458.

[9]. S. M. Dankwardt, R. L. Martin, C .S. Chan, H. E. Van Wart, K. A. M. Walker, N. G. Delaet, L. A. Robinson, Bioorg. Med. Chem. Lett. 2001, II, 1465-1468.

[10]. Y. K. Agrawal, H. Kaur, React. Funct. Polym. 1999, 39, 155-164.

[11]. Y. K. Agrawal, S. A. Patel, Rev. Anal. Chem. 1980, 4, 237-276.

[12]. F. Huguet, A. Melet, R. A. de Sousa, A. Lieutaud, J. Chevalier, L. Maigre, P. Deschamps, A. Tomas, N. Leulliot, J. M. Pages, I. Artaud, Chem.Med.Chem. 2012, 7, 1020-1030.

[13]. C. Lee, E. Choi, M. Cho, B. Lee, S. J. Oh, S. K. Park, K. Lee, H. M. Kim, G. Han, Bioorg. Med. Chem. Lett. 2012, 22, 4189-4192

[14]. M. A. Santos, S. M. Marques, T. Tuccinardi, P. Carelli, L. Panelli, A. Rossello, Bioorg. Med. Chem. 2006, 14, 7539- 7550.

[15]. J. I. Levin Curr. Top. Med. Chem. 2004, 4, 1289-1310.

[16]. A. V. Malkov, Z. Bourhani, P.Kočovský, Org. Biomol. Chem., 2005,3, 3194-3200.

[17]. Pasha M.A., Jayashankara V.P., Venugopala K.N., and Rao G.K., Zinc Oxide (ZnO): an efficient catalyst for the synthesis of 4arylmethylidene-2-phenyl-5-(4H)-oxazolones having antimicrobial activity, Journal of Pharmacology and Toxicology, 2007, 2, 264270 . 